\title{
Multi-disciplinary Conceptual Design Knowledge of Multi-stage Hybrid Rocket Using Data Mining Technique
}

\author{
Masahiro Kanazaki ${ }^{1}$, Kazuhisa Chiba ${ }^{2}$, Koki Kitagawa ${ }^{3}$, Toru Shimada ${ }^{3}$ and Masashi Nakamiya ${ }^{4}$ \\ 1. Department of Aerospace Engineering, Graduate School of System Design, Tokyo Metropolitan University, Tokyo 191-0065, \\ Japan \\ 2. Graduate School of Engineering, Hokkaido University of Science, Sapporo 006-8585, Japan \\ 3. Institute of Space and Astronautical Science, Japan Aerospace Exploration Agency, Sagamihara 252-5210, Japan \\ 4. Research Institute for Sustainable Humanosphere, Kyoto University, Kyoto 606-8501, Japan
}

Received: November 18, 2014 / Accepted: December 01, 2014 / Published: January 25, 2015.

\begin{abstract}
This paper deals with the application of data mining techniques to the conceptual design knowledge for a LV (launch vehicle) with a HRE (hybrid rocket engine). This LV is a concept of the space transportation, which can deliver micro-satellite to the SSO (sun-synchronous orbit). To design the higher performance LV with HRE, the optimum size of each component, such as an oxidizer tank containing liquid oxidizer, a combustion chamber containing solid fuel, a pressurizing tank and a nozzle, should be acquired. The Kriging based ANOVA (analysis of variance) and SOM (self-organizing map) are employed as data mining techniques for knowledge discovery. In this study, the paraffin (FT-0070) is used as a propellant of HRE. Then, the relationship among LV performances and design variables are investigated through the analysis and the visualization. To calculate the engine performance, the regression rate is computed based on an empirical expression. The design knowledge is extracted for the design knowledge of the multi-stage LV with HRE by analysis using ANOVA and SOM. As a result, the useful design knowledge on the present design problem is obtained to design HRE for space transportation.
\end{abstract}

Key words: Hybrid rocket, data mining techniques, multidisciplinary design.

\section{Introduction}

The kind of rocket presently used for space transportation is either a solid rocket or liquid rocket (Fig. 1). The HRE (hybrid rocket engine) is a different type of rocket that uses a liquid oxidizer and a solid fuel. This rocket has advantages of being high safe, low cost and environment-friendly. Therefore, there are expectations for the HRE as a safe and green means of propulsion for future space transport. The HRE was successfully put to practical use for Space-Ship One [1], which completed the first private manned space flight.

On the other hand, the most serious problem of the

Corresponding author: Masahiro Kanazaki, research fields: Ph.D., associate professor, research fields: aeronautics, aerodynamic design, computational fluid dynamics, design optimization. E-mail: kana@sd.tmu.ac.jp.
HRE as a form of space transportation is the low fuel regression rate which is the melting rate of the solid fuel. Due to the low regression rate, if the engine design is not appropriate, the thrust of the HRE will be insufficient compared with that of the solid rocket and liquid rocket engines. The thrust of the HRE is affected by the mass flow of the vaporized fuel. The mass flow of vaporized fuel is decided by the oxidizer mass flow, the fuel grain length and the inner radius of the fuel grain port. If these parameters are combined optimally, the thrust will be sufficient. Since these parameters also decide the engine geometry, the weight and trajectory are also affected. As a result, knowledge discovery techniques are desirable for a multi-disciplinary design of an HRE for a LV (launch vehicle).

A previous study [2] developed a MDO 
(multi-disciplinary optimization) methodology that includes a technique for an empirical-model-based evaluation of the performance of a LV with an HRE, and optimized several kinds of launch vehicles with an HRE using a GA (genetic algorithm). In addition, design knowledge discoveries have been also carried out by means of several knowledge discovery techniques for a single-stage sounding rocket [3-5]. In this study, the developed evaluation method used to discover the design knowledge of a three-stage LV with HRE (Fig. 2a), can deliver 10.0-50.0 kg class micro-satellites, which used for scientific observation of the Earth, to a SSO (sun-synchronous orbit) which is $800 \mathrm{~km}$ altitude. A SSO is a geocentric orbit which combines altitude and inclination in such a way that an object on that orbit ascends or descends over any given point of the Earth's surface at the same local mean solar time. Fig. $2 b$ shows the image of the engine designed in this study. Paraffin fuel (FT-0070) [6] is employed as a fuel, LOX (liquid oxygen) is employed as an oxidizer, and the combustion type is a swirling-oxidizer-type engine. To obtain the quantitative information, an ANOVA (analysis of variance) is applied. To visualize the design problem, a SOM (self-organizing map) is employed.

This paper is organized as follows: Section 2 presents the performance evaluation procedure for $\mathrm{LV}$ using HRE; Section 3 describes methodologies for the design knowledge discovery techniques such as ANOVA and SOM; Section 4 defines the design problem; Section 5 shows the results of the knowledge discovery of three-stage hybrid rocket; and Section 6 gives the conclusions.

\section{Performance Evaluation for $\mathrm{LV}$ with $\mathrm{HRE}$}

This study deals with the LV of a three-stage rocket with a chamber, an oxidizer tank, a pressurizing tank, a nozzle and a payload (Fig. 2b) that has an HRE. Fig. 3 shows the evaluation procedure proposed in the previous study [2].

Generally, the regression rate in the radial direction of the fuel is expressed as follows:

$$
\underset{\text { port }}{\&}(t)=a \cdot G_{\text {oxi }}^{n}(t)
$$

Eq. (1) is empirically defined and the coefficient $a$

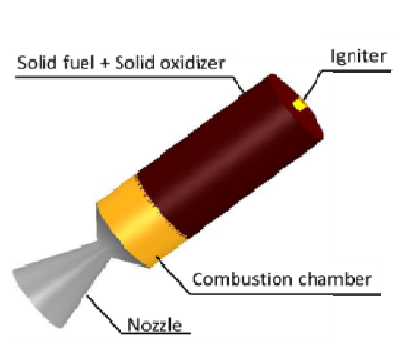

(a)

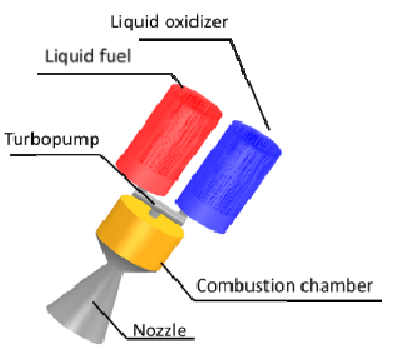

(b)
Fig. 1 Schematic illustrations of rocket engine which widely used for the space transportation: (a) solid rocket engine and (b) liquid rocket engine.

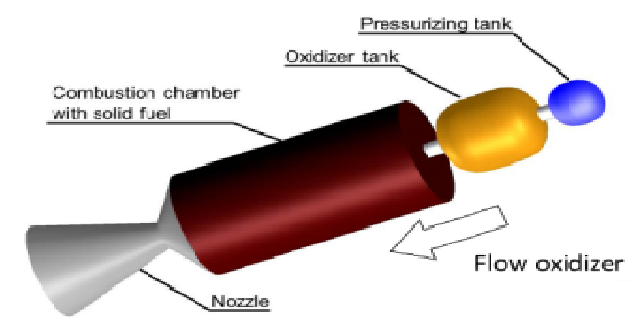

(a)

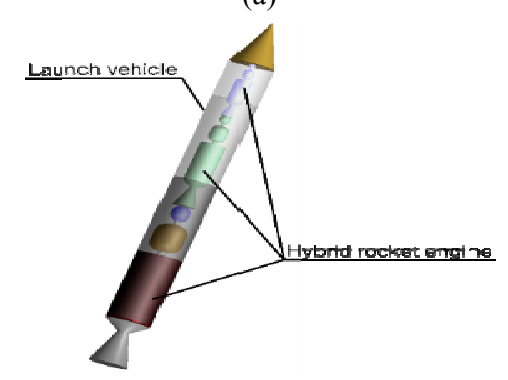

(b)

Fig. 2 Conceptual illustration of the $L V$ considered in this study: (a) the HRE and (b) the three-stage $L V$ with an HRE.

and index $n$ are generally decided from the experiments for fuels with a single port $[2,6]$. The HRE considered here supplies the swirling oxidizer into the WAX (FT-0070) fuel. $a$ and $n$ in Eq. (1) are determined from the experiment for a non-swirling oxidizer with the WAX fuel [6]; then, Eq. (1) can be written as:

$$
\underset{p o r t}{\&}(t)=0.1561 \times 10^{-3} \cdot G_{o x i}^{0.3905}(t)
$$

In this study, a swirling-oxidizer-type HRE, which 
can achieve a higher regression rate [7], is assumed. For this purpose, the empirical multiplication with the coefficient of Eq. (2) is carried out.

$$
\begin{aligned}
& \underset{\mathbb{p}_{\text {port } m} m}{\&_{-}}(t)=a_{-m} \cdot G_{\text {oxi_m }}^{0.3905}(t) \\
& \left(a_{-m}=\alpha 0.1561 \times 10^{-3}\right)
\end{aligned}
$$

In this study, this coefficient, $a_{-} m$, is a part of the design variables that determine the strength of the oxidizer swirl. The range of $\alpha$ used to decide the design range of $a_{-} m$ is from 4 to 10 . The estimation methods for the engine size and performance are presented in the following sections.

\subsection{Grain Configuration}

The combustion chamber considered in this study contains solid fuel with a single port to supply the oxidizer. $r_{\text {port_ } \_}(0)$ and $L_{\text {fuel } \_} m$ are calculated for each stage as follows:

$$
\begin{aligned}
& r_{p o r r_{-} m}(0)=\sqrt{\frac{n \&_{o x i} \mathbb{K}_{-}(0)}{\pi G_{o x i} m}(0)}
\end{aligned}
$$

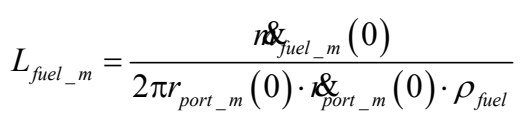

Here, $n s_{\text {uel } \_} m(0)$ is obtained from the definition of

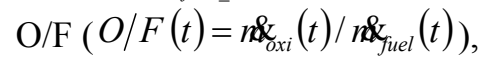

$$
n \delta_{\text {juel } \_m}(0)=\frac{n \&_{o x i} m}{O / F_{-m}(0)}
$$

$n \&_{o x i} m(0), G_{\text {oxi_ } m}(0)$, and $O / F_{-}(0)$ are part of the design parameters listed in the developed evaluation module.

\subsection{O/F and Chamber Pressure Evaluation}

$\mathrm{O} / \mathrm{F}$ at time $t$ is calculated as the absolute value of $r_{s i}(n)$ can be written as:

$$
O / F_{-m}(t)=\frac{n \&_{0 x i} m}{n \delta_{\text {jiel } m}(t)}
$$

$n x_{\text {juel } \_m}(t)$ is estimated based on $\&_{\text {port } m} \&_{m}(t)$ obtained from Eq.(3) as follows:

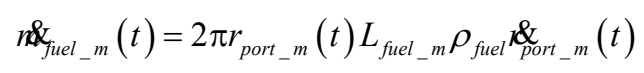

$P_{c h m}$ at time $t$ is calculated as:

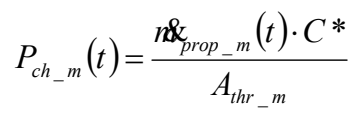

Here, $n \&_{p r o p_{-} m}(t)$ is obtained by adding $n \aleph_{x x i-m}(t)$ and $n k_{\text {juel }-m}(t) . C^{*}(t)$ is obtained from $O / F_{-} m(t-\Delta t)$, $P \_m(t-\Delta t)$ and $\varepsilon_{\_} m$ using the NASA-CEA (National Aeronautics and Space Administration-Chemical Equilibrium with Applications) program [8].

\subsection{Weight Estimation}

In this study, LOX is employed as an oxidizer and WAX (FT-0070) is used as the fuel. The required oxidizer mass, $M_{\text {oxi } m} m$, and fuel mass, $M_{\text {furl_ } m} m$, are calculated for each stage as follows:

$$
\begin{aligned}
& M_{o x i_{-} m}=\int_{0}^{t c_{-} m} \dot{m}_{o x i_{-} m}(t) \mathrm{d} t \\
& M_{\text {fuel } \_m}=\int_{0}^{t c_{-} m} \dot{m}_{f u e l_{-} m}(t) \mathrm{d} t
\end{aligned}
$$

Here, $t_{c_{-} m}$ is one of the design parameters. Helium gas is used as the pressurizing gas. The mass of the pressurizing gas required is obtained by solving the state equation as follows:

$$
P_{p t_{-} m}(0) \mathrm{VOl}_{\text {pre } m}=M_{H e_{-} m} \mathrm{RTi}
$$

Assuming that $P_{p t_{-} m}$ is equal to $P_{o t_{-} m}$ when the supply of helium gas is stopped, the state equation can be expressed as follows:

$$
P_{o t_{-} m}\left(t c_{-} m\right)\left(V o l_{p r e \_} m+V o l_{\text {res } \_m}\right)=M_{H e_{-} m} R T f
$$

Eliminating $V_{o l} l_{o t}$ from Eqs. (12) and (13), $M_{H_{-} m}$ can be calculated. The temperature of helium after combustion, i.e., $T_{f}$, is calculated as:

$$
T f=T i\left(\frac{P_{o t_{-} m}(t c)}{P_{p t_{-} m}(0)}\right)^{\frac{\kappa-1}{\kappa}}
$$

The initial helium temperature $T_{i}$ is $273 \mathrm{~K}$, and its $\kappa$ is 1.66 . In this study, $P_{p t_{-} m}(0)$ is one of the design variables.

The structure of the engine is assumed to be the same as that of the solid rocket M-V [9]. In this study, the combustion chamber and the pressurizing tank are 
assumed to be made of CFRP (carbon-fiber-reinforced plastic) to reduce the structural weight. The thicknesses of the shells used for the chamber and the tank are calculated by assuming that $\sigma_{c h}$ and $s f$ values for these tanks are $2.4 \mathrm{GPa}$ and $1.5 \mathrm{GPa}$ [2], respectively. $M_{c h \_m}$ and $M_{p t_{-} m}$ are calculated using:

$$
\begin{aligned}
M_{c h_{-} m} & =\frac{P_{c h_{-} m} V_{c o l} l_{c L_{-} m}}{17.3 \times 10^{4}} \\
M_{p t_{-} m} & =\frac{P_{p t_{-} m} V_{0 t_{-} m}}{17.3 \times 10^{4}}
\end{aligned}
$$

Here, the denominator on the right-hand side is the measure of the structural performance. In this study, the same values as those used in Ref. [9] have been used in Eqs. (15) and (16). The oxidizer tank is assumed to be made of CFRP with an aluminum liner, which can prevent microcracks at extremely low temperatures. This tank has a $0.05 \mathrm{~m}$ thick heat-insulating material. The thickness of the shell used for the tank is calculated by assuming that $\sigma_{o t}$ and $s f$ are set to $2.4 \mathrm{GPa}$ and $1.5 \mathrm{GPa}$ [2], respectively. The LOX tank used in this study is assumed to have a structure similar to the conventional the liquid helium (LHe2) tank. In this study, $P_{\text {ot } \_}(0)$ is twice of $P_{\text {ch } m}$. $M_{o t_{-} m}$ is calculated as follows:

$$
\begin{aligned}
M_{o t_{-} m} & =\frac{P_{o t_{-} m}(0) \mathrm{Vol}_{o t_{-} m}}{4.4 \times 10^{4}} \\
= & \frac{2 P_{c h_{-} m}(0) \mathrm{Vol}_{o t_{-} m}}{4.4 \times 10^{4}}
\end{aligned}
$$

Here, $P_{o t \_} m(0)$ and $P_{p t \_}(0)$ are design variables. In this study, the length $\left(L_{c h \_} m, L_{p t_{-} m}\right.$ and $\left.L_{o t_{-} m}\right)$, diameters,

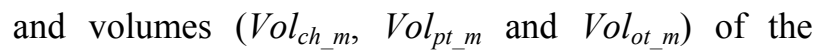
chamber and tanks of the designed engine are determined using the same calculation procedure as that used in Ref. [2].

An empirical equation [10] as expressed by Eq. (18), is used to calculate $M_{n o z} m$ :

$$
M_{n o z_{-} m}=125.0\left(\frac{M_{\text {prop } \_m}}{5,400.0}\right)^{\frac{2}{3}}\left(\frac{\varepsilon_{-m}}{10.0}\right)^{\frac{1}{4}}
$$

Here, $M_{\text {prop } \_} m$ is obtained by adding $M_{\text {oxi } m}$ and $M_{\text {fuel } \_m} . \varepsilon_{-} m$ is one of the design parameters.
Using an empirical equation [10], the mass of other equipment (an injector, an igniter, ducts and control devices) is found to be approximately $30 \%$ of the total structural mass of the engine $M_{\text {tot }} m$. Therefore, the total mass of the $m$-th stage is expressed by Eq. (19) as:

$$
\begin{aligned}
& M_{\text {tot } \_}=M_{p a y_{-} m}+M_{p r o p_{-} m}+M_{H_{e_{-} m} m} \\
& \quad+1.3\left(M_{c h_{-} m}+M_{o t_{-} m}+M_{p t_{-} m}+M_{n o z_{-} m}\right)
\end{aligned}
$$

The rocket length $L_{\text {tot_ }-m}$ is calculated by taking the sum total of $L_{c h \_}, L_{p t \_}, L_{o t_{-} m}$ and $L_{n o z_{-} m}$. In order to load the payload on the $3 \mathrm{rd}$ stage, $L_{\text {tot } 3}$ is multiplied by the coefficient 1.5 .

\subsection{Trajectory Estimation}

This study assumes the rocket to be a mass point from the time of launch to the target orbit. Its equations of motion are expressed as:

$$
\begin{gathered}
\frac{\mathrm{d} r}{\mathrm{~d} t}=V \sin \gamma \\
\frac{\mathrm{d} \theta}{\mathrm{d} t}=\frac{V \cos \gamma \cos \psi}{r \cos \phi} \\
\frac{\mathrm{d} \phi}{\mathrm{d} t}=\frac{V \cos \gamma \sin \psi}{r} \\
\frac{\mathrm{d} V}{\mathrm{~d} t}=\frac{T h_{-m}-D}{M}-g \sin \gamma \\
\frac{\mathrm{d} \gamma}{\mathrm{d} t}=\left(\frac{V}{r}-\frac{g}{V}\right) \cos \gamma \\
\frac{\mathrm{d} \psi}{\mathrm{d} t}=-\frac{V \cos \gamma \tan \phi \cos \psi}{r}
\end{gathered}
$$

$T_{h \_\mathrm{m}}$ is calculated as:

$$
T h_{-} m=\eta_{C F} \eta_{C^{*}}\left(n \alpha_{p r o p_{-} m} u e_{-} m+\left(P_{e_{-} m}-P_{a}\right) A_{e_{-} m}\right)
$$

In this study, $\eta_{C F}$ is assumed to be 0.98 and $\eta_{C^{*}}$ is assumed to be 0.95 in magnitude $[9,10] . u_{e_{-} m}$ and $P_{e_{-} m}$ can be obtained from $O / F_{-} m(\mathrm{t}), P_{c h \_} m(\mathrm{t})$ and $\varepsilon_{-} m$ using NASA-CEA program.

The drag estimation is based on the flight data of JAXA's solid rocket S-520 [11]. Fig. 4 shows the 
variation in $C_{D, S-520}$ with the Mach number. In this study, the data presented in Fig. 4 has been used. $C_{D f}$ is empirically estimated by assuming that the outer surface of the rocket is a flat plate [12].

$$
C_{D f}=\frac{0.455}{\left(\log _{10} R e\right)^{2.58}\left(1+0.144 M^{2}\right)^{0.65}}
$$

The Reynolds number is calculated based on the rocket's length. $C_{D p}$ is estimated as:

$$
C_{D p}=C_{D, S-520}-C_{D f, S-520} \frac{S_{w e t, s-520}}{S_{\text {wet }, \text { design }}}
$$

Here, $C_{D, S-520}$ is obtained from the measured flight data of S-520, and $C_{D f, S-520}$ is calculated using Eq. (27). The second term of Eq. (28) is $C_{D f \text {, design. The total }}$ drag is obtained by adding the pressure drag and the friction drag.

$$
D=q\left(S_{\text {wet,design }} C_{D f}+S_{\text {cro,design }} C_{D p}\right)
$$

Since the pressure drag and the friction drag are estimated separately, the effect of the rocket's aspect ratio on its aerodynamic performance can be evaluated.

\section{Methodologies for Design Knowledge Discovery}

The optimization procedure design consists of the following steps. First, $N$ design samples are selected by LHS (Latin hypercube sampling) [13, 14], which is a space filling method, and then assessed for the construction of an initial Kriging surrogate model. Second, ANOVA is applied to evaluate effects of design variables to the objective functions. Finally, SOM is applied to visualize the design problem. The procedure is illustrated in Fig. 5.

\subsection{ANOVA}

ANOVA, a method for multivariate analyses, is carried out to differentiate the contribution of different design variables to the variance of the responses from the global model. Specifically, to evaluate the

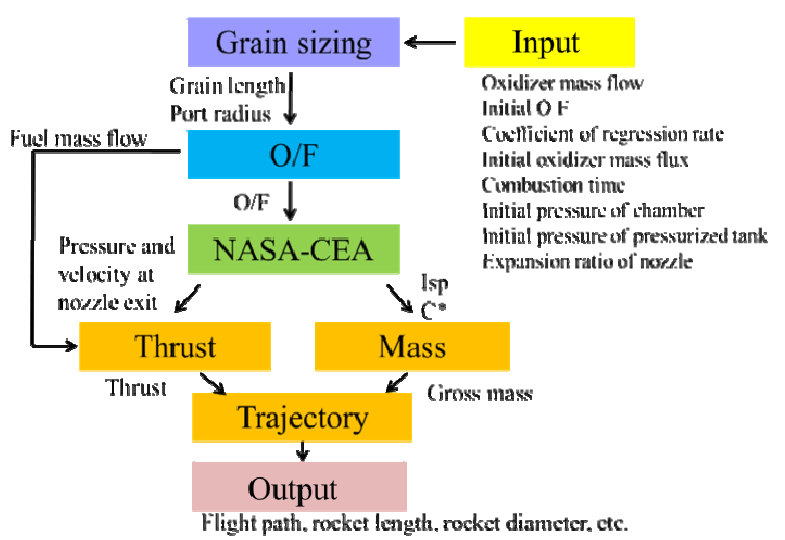

Fig. 3 Flowchart of the performance evaluation of the $\mathrm{LV}$ with an HRE [2].

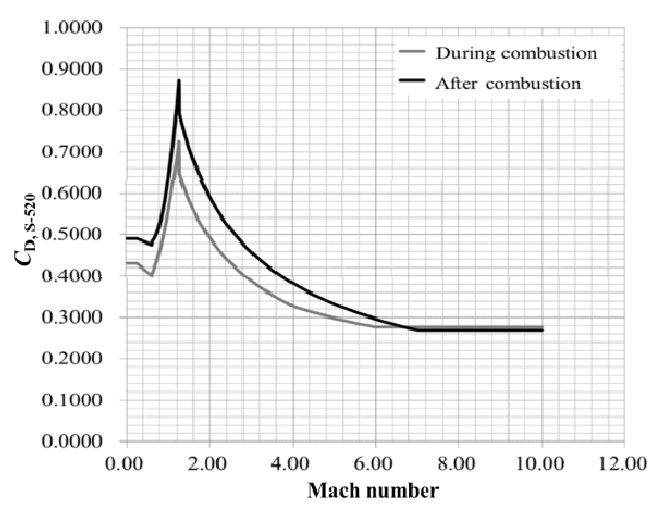

Fig. 4 Mach number vs. $C_{D, S-520}$ measured in the flight test of the solid rocket $S-520[2,11]$.

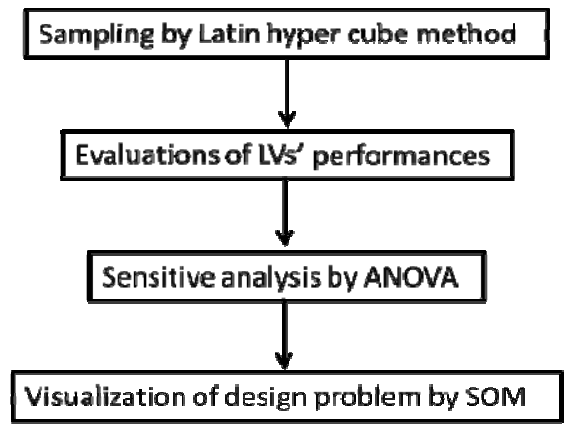

Fig. 5 Procedure of design knowledge discovery.

contribution of each design variable, the total variance of the model is decomposed into the variance attributed to each design variable and the interactions between each other. The decomposition is accomplished by integrating variables out of the model $\hat{y}$. The variance of design variable $x_{i}$ to $\mu$ is defined as:

$$
\mu_{i}\left(x_{i}\right) \equiv \int \ldots \int \hat{y}\left(x_{1}, \ldots . ., x_{n}\right) \mathrm{d} x_{1}, \ldots, \mathrm{d} x_{i-1}, \mathrm{~d} x_{i+1}, . ., \mathrm{d} x_{n}-\mu
$$


where, the total mean $\mu$ is calculated as:

$$
\mu \equiv \int \cdots \int \hat{y}\left(x_{1}, \ldots, x_{n}\right) \mathrm{d} x_{1}, \ldots, \mathrm{d} x_{n}
$$

The proportion of the variance attributed to the design variable $x_{i}$ to the total variance of the model can be expressed as:

$$
p \equiv \frac{\int\left[\mu_{i}\left(x_{i}\right)\right]^{2} \mathrm{~d} x}{\int \cdots \int\left[\hat{y}\left(x_{1}, \ldots, x_{n}\right)-\mu\right]^{2} \mathrm{~d} x_{1} \ldots \mathrm{d} x_{n}}
$$

The value obtained by Eq. (32) indicates the sensitivity of an objective function to the variance of a design variable.

\section{$3.2 S O M$}

SOM [13-16] is an unsupervised learning, nonlinear projection algorithm from high to low dimensional space. This projection is based on self-organization of a low-dimensional array of neurons. The lattice of the gird can be either hexagonal or rectangular to generate SOM. In this paper, the former is used because it is more pleasing to the eye.

Each neuron $k$ on the map is represented by an $n$-dimensional prototype vector $m_{k}=\left(m_{k 1}, m_{k 2}, \ldots\right.$, $\left.m_{k n}\right)$, where $n$ is the dimension of the design space, that is number of design variables and objective functions. To train the map, input vector $\boldsymbol{X}$ which represents a sampling design is selected and the nearest neuron $m_{c}$ (the best matching unit, BMU) is found from the prototype vectors on the map. The prototype vectors of the $m_{c}$ and its neighbors on the grid $m_{k}$ are moved towards $\boldsymbol{X}$ as follows:

$$
m_{k}=m_{k}+\alpha(t)\left(\boldsymbol{X}-m_{k}\right)
$$

where, $\alpha(t)$ is learning rate and it decreases monotonically with time. This process as shown in Fig. 6 is iterated until $\alpha(t)$ is converged well.

During the iterative training, prototype vectors are also converged. The closer two patterns are in the original space, the closer is the response of two neighboring neurons in the low-dimensional map. Thus, SOM reduces the dimension of input data while preserving their features. The trained SOM is systematically converted into visual information, and qualitative information can be obtained.

In this study, commercial software modeFrontier ${ }^{\circledR}$ is used for visualization. modeFrontier ${ }^{\circledR}$ creates a map in a two-dimensional hexagonal grid, and this map can be colored based on the every attribute values (that is, design variables, and objective functions). Therefore, $n$ component planes are created and can be compared visibly. However, if $n$ is large number and it is not pleasing to the eye, component planes should be arranged for the efficient comparison. In this study, component plane reorganization was used together with traditional correlation analysis [16]. Correlations between component pairs are revealed as similar patterns in identical positions of the component plane so called "SOM's SOM". Pattern matching is something that the human eye is very good at. The knowledge management can be made even easier if the component planes are reorganized so that correlated ones are possibly presented near each other.

\section{Formulation}

In this study, a three-stage LV with an HRE, which can deliver micro-satellites to the SSO (apogee $=800$ $\mathrm{km}$ ), is considered. Fig. 7 shows the assumed flight profile. The rocket is launched toward the south at an $89^{\circ}$. launch angle. The 2 nd stage is immediately ignited after the combustion of the first stage is completed. The 3rd stage coasts along the oval orbit after the combustion of the 2nd stage is completed.

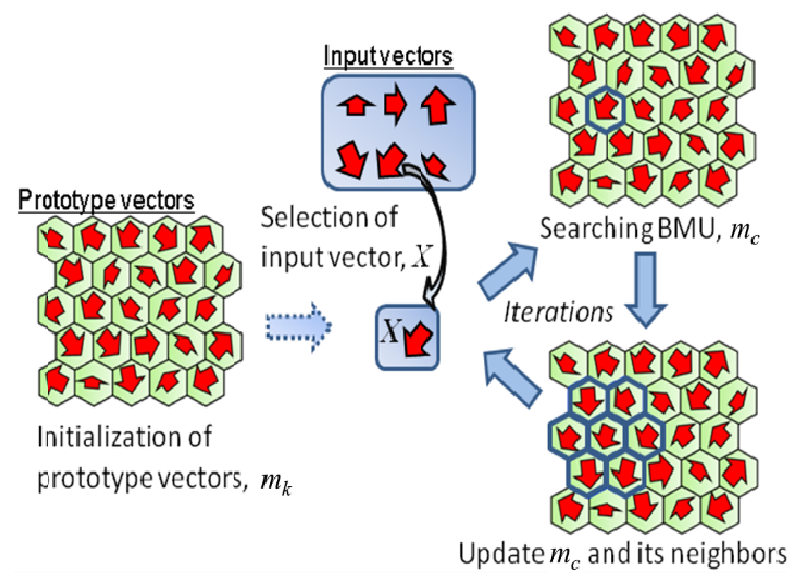

Fig. 6 Procedure of SOM training. 
After this coasting, the 3 rd stage is ignited. $t_{\text {coast }}$, as shown in Fig. 7, is also one of the design variables.

The design variables and their ranges are listed in Table 1. In common aerospace vehicle design problems, the gross weight of a vehicle should be minimized. Additionally, in this study, the ratio of the payload weight to the drag weight is offset by the operation cost of the rocket. Thus, total weight, $M_{\text {tot }}$, $M_{p a y}$ and $M_{p a y} / M_{t o t}$, are evaluated for knowledge discovery.

The trajectory constraints assumed are as follows:

- The flight altitude is over $250 \mathrm{~km}$ after the combustion of the 3rd stage;

- The angular momentum is more than $52,413.5$ $\mathrm{kg} \cdot \mathrm{km}^{2} / \mathrm{s}$ after the combustion of the $3 \mathrm{rd}$ stage in order to ensure that the rocket reaches $800 \mathrm{~km}$ at the apogee;

- The flight path angle after combustion of the $3 \mathrm{rd}$ stage is between $\pm 5.0^{\circ}$.

The constraints for the structure are as follows:

- The aspect ratio of the rocket is less than 20.0;

- The diameter of the nozzle exit is less than that of all stages;

- The area of the grain port is more than twice the nozzle throat area of all stages.

\section{Results and Discussion}

\subsection{Result of ANOVA}

Fig. 8 shows the ANOVA visualization results.

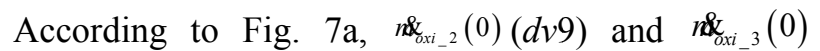
$(d v 17)$ are important parameters for improving $M_{\text {pay }} / M_{\text {tot }}$. A heavier payload can be launched with a larger engine in the 1st stage. However, the 2nd and 3rd stages have to be carefully designed to ensure a more efficient launch. According to Fig. 7b, $n_{0 \times x i 1}(0)$ $(d v 1)$ has the dominant effect on $M_{t o t} . t_{c-1}(d v 5)$ and $d v 9$ also influence $M_{t o t}$. They determine the total mass of the fuel and oxidizer and that the 1st stage should be heavier.

\subsection{Result of SOM}

Fig. 9a shows the SOM visualization results as
Table 1 Design space ( $d v 1-d v 8$ are for 1st stage, $d v 9-d v 16$ are for 2 nd stage and $d v 17-d v 25$ is for 3rd stage).

\begin{tabular}{|c|c|c|c|}
\hline \multicolumn{2}{|c|}{ Design variable } & Lower & Upper \\
\hline$d v 1$ & $n_{b x i_{i} 1}(0)(\mathrm{kg} / \mathrm{s})$ & 50.0 & 150.0 \\
\hline$d v 2$ & $O / F_{-}(0)(-)$ & 2.0 & 3.0 \\
\hline$d v 3$ & $a_{-1}\left(\times 10^{-3}\right)$ & 0.39025 & 1.0927 \\
\hline$d v 4$ & $G_{\text {oxi__ }}(0)\left(\mathrm{kg} / \mathrm{m}^{2} \mathrm{~s}\right)$ & 200.0 & 400.0 \\
\hline$d v 5$ & $t_{c_{-} 1}(\mathrm{~s})$ & 40.0 & 60.0 \\
\hline$d v 6$ & $P_{c h \_1}(0)(\mathrm{MPa})$ & 0.5 & 3.0 \\
\hline$d v 7$ & $P_{p t_{-} 1}(0)(\mathrm{MPa})$ & 15.0 & 40.0 \\
\hline$d v 8$ & $\varepsilon_{-1}(-)$ & 2.0 & 8.0 \\
\hline$d v 9$ & $n \&_{o x i_{-}}(0) \quad(\mathrm{kg} / \mathrm{s})$ & $0.1 n_{o x i_{-} 1}^{\&_{1}}(0)$ & $(1 / 6) n \mathbb{\alpha}_{\alpha x i} \mathbb{R}_{-1}(0)$ \\
\hline$d v 10$ & $O / F_{-2}(0)(-)$ & 2.0 & 3.0 \\
\hline$d v 11$ & $a_{-2}\left(\times 10^{-3}\right)$ & 0.6244 & 1.561 \\
\hline$d v 12$ & $G_{\text {oxi_ }}(0)\left(\mathrm{kg} / \mathrm{m}^{2} \mathrm{~s}\right)$ & 10.0 & 200.0 \\
\hline$d v 13$ & $t_{c_{-} 2}(\mathrm{~s})$ & $t_{c_{-} 1}+30.0$ & $t_{c_{-} 1}+50.0$ \\
\hline$d v 14$ & $P_{c h \_2}(0)(\mathrm{MPa})$ & 0.5 & 2.0 \\
\hline$d v 15$ & $P_{p t_{-} 2}(0)(\mathrm{MPa})$ & 10.0 & 30.0 \\
\hline$d v 16$ & $\varepsilon_{-2}(-)$ & 20.0 & 50.0 \\
\hline$d v 17$ & $n_{\alpha x i_{-} 3}^{K_{3}}(0) \quad(\mathrm{kg} / \mathrm{s})$. & $0.1 n_{o x i-2}^{\delta_{-}}(0)$ & $0.2 n \&_{o x i_{-}}(0)$ \\
\hline$d v 18$ & $O / F_{-3}(0)(-)$ & 2.0 & 3.0 \\
\hline$d v 19$ & $a_{-3}\left(\times 10^{-3}\right)$ & 0.6244 & 1.4049 \\
\hline$d v 20$ & $G_{o x i} 3(0)\left(\mathrm{kg} / \mathrm{m}^{2} \mathrm{~s}\right)$ & 10.0 & 120.0 \\
\hline$d v 21$ & $t_{c \_3}(\mathrm{~s})$ & $t_{c_{-} 2}+20.0$ & $t_{c_{-} 2}+20.0$ \\
\hline$d v 22$ & $P_{c h \_} 2(0)(\mathrm{MPa})$ & 0.1 & 1.0 \\
\hline$d v 23$ & $P_{p t \_}(0)(\mathrm{MPa})$ & 15.0 & 40.0 \\
\hline$d v 24$ & $\varepsilon_{-3}(-)$ & 40.0 & 70.0 \\
\hline$d v 25$ & $t_{\text {coast }}(\mathrm{s})$ & 140.0 & 180.0 \\
\hline
\end{tabular}

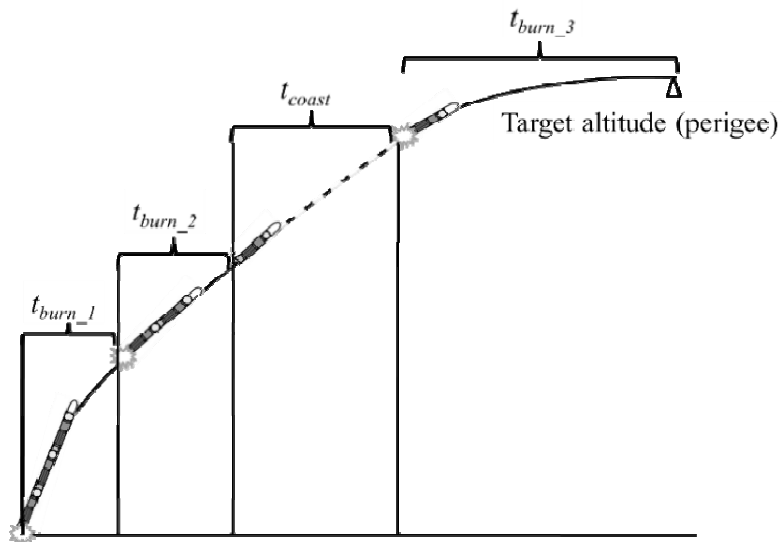

Fig. 7 Flight of the designed rocket.

maps colored by each attribute value (design variables and objective functions), and they are clustered based on the similarity in color patterns. According to the 
maps, $d v 1$ is similar to $M_{t o t}$, and $d v 17$ is similar to $M_{\text {pay }} / M_{\text {tot }}$. These results suggest that they are strongly related each other. This result agrees with the ANOVA visualization results. Fig. $9 \mathrm{~b}$ compares trained maps colored by $M_{p a y} / M_{t o t}, M_{t o t}$, and $M_{p a y}$. These maps show a tradeoff between the two design

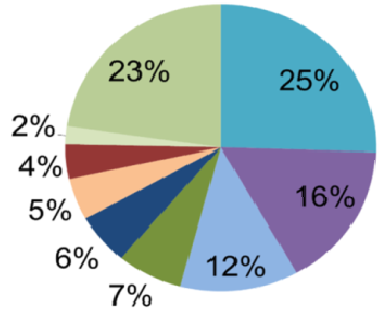

(a) objectives. In addition, the SOM unit showed a heavy $M_{\text {pay }}$ but did not always show a high $M_{\text {pay }} / M_{\text {tot }}$. This result suggests that $M_{p a y} / M_{t o t}$ is a reasonable design objective function with regard to the rocket efficiency
Fig. 8 Visualization with ANOVA: (a) sensitivity to $M_{p a y} / M_{t o t}$ and (b) sensitivity to $M_{t o t}$.

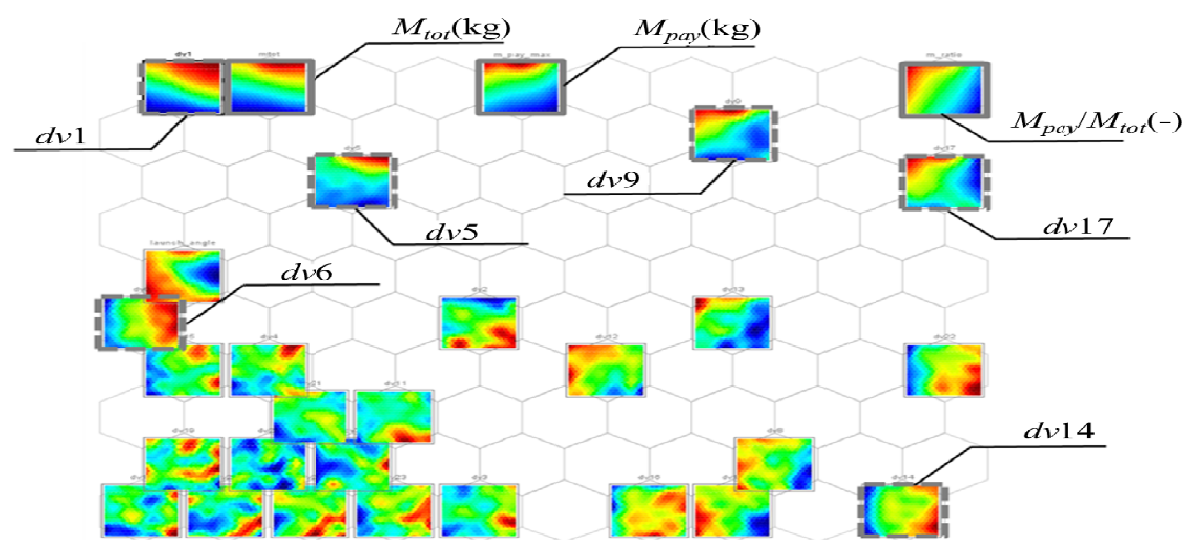

(a)

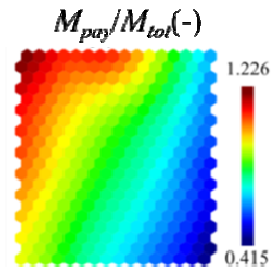

$M_{\text {fol }}(\mathrm{kg})$
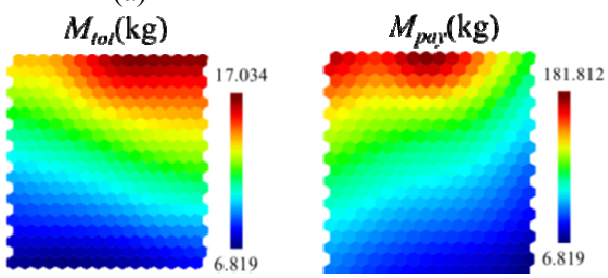

(b) $d v 1(\mathrm{~kg} / \mathrm{s}$.

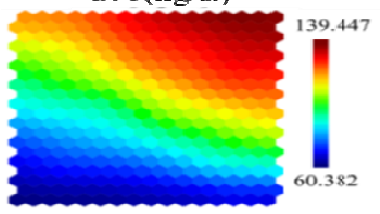

$d v 9(\mathrm{~kg} / \mathrm{s}$.

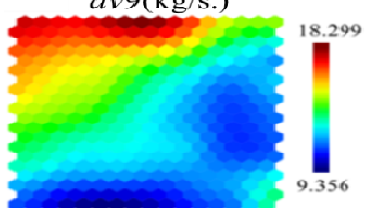

$d v 5(\mathrm{~s}$.

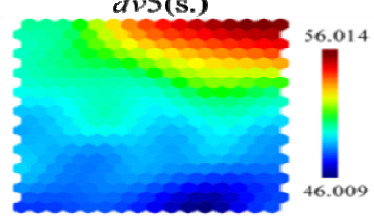

$d v 14(\mathrm{MPa})$

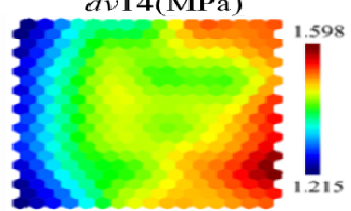

(c)

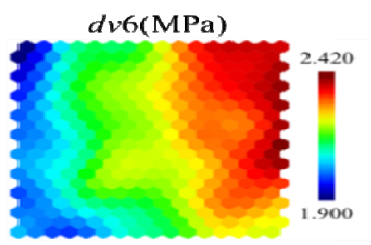

$d v 17(\mathrm{~kg} / \mathrm{s}$.

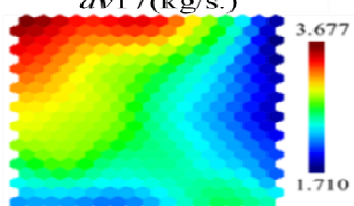

- $d v 1-d v 6$

= $d v 5$

밀 $d v 9$

a $d v 6$

$=d v 1-d v 5$

others

(b) 
for a rocket to deliver a heavier payload. Fig. 9c shows maps colored with design variables. Here, $d v 1$, $d v 5, P_{c h \_} 1(0)(d v 6), d v 9, P_{c h \_} 3(0)(d v 14)$, and $d v 17$ were selected according to the ANOVA results (Fig. 7) and correlation among attribute values (Fig. 8a) According to Figs. $9 \mathrm{~b}$ and $9 \mathrm{c}, d v 5, d v 6$ and $d v 14$ should be smaller, and $d v 9$ and $d v 17$ should be larger. Remarkably, $d v 1$ did not need to be maximized for the design of an efficient LV (i.e., high $\left.M_{p a y} / M_{t o t}\right)$. Because an excessively large or small $d v 1$ does not allow for a high $M_{p a y} / M_{t o t}$, it should be carefully determined.

\section{Conclusions}

In this study, knowledge discovery techniques were used to discover the design knowledge for an LV with an HRE that delivers micro-satellites to an SSO. The launch vehicle performances (the flight, the weight and the propulsion) were evaluated based on empirical model. The evaluated functions were to the total mass of the rocket, the maximum payload mass and to maximize the ratio of the payload mass to the total mass in terms of the cost. The results were visualized by ANOVA and SOM. ANOVA result showed that the oxidizer mass flow of every stage is very important. The oxidizer mass flow at the 1st stage has predominant effect to the vehicle total mass. On the other hand, the oxidizer mass flow at the 2nd and 3rd stages effective to the payload and total mass ratio. SOM result suggested that a tradeoff between $M_{p a y} / M_{t o t}$, and $M_{t o t}$. In addition, the relationship among design variables and evaluated performances could be visualized.

\section{References}

[1] Thicksten, Z., Macklin, F., and Campbell, J. 2008. "Handling Considerations of Nitrous Oxide in Hybrid Rocket Motor Testing." Presented at the 44th AIAA/ASME/SAE/ASEE Joint Propulsion Conference \& Exhibit, Hartford, CT.

[2] Kosugi, Y., Oyama, A., Fujii, K., and Kanazaki, M. 2011. "Multidisciplinary and Multi-objective Design Exploration Methodology for Conceptual Design of a Hybrid Rocket." Presented at the International IEEE
Aerospace conference, St. Louis, Missouri.

[3] Chiba, K., Kanazaki, M., Nakamiya, M., Kitagawa, K., and Shimada, T. 2014. "Diversity of Design Knowledge for Launch Vehicle in View of Fuels on Hybrid Rocket Engine." Journal of Advanced Mechanical Design, Systems, and Manufacturing 8 (3): 1-14.

[4] Chiba, K., Kanazaki, M., Nakamiya, M., Kitagawa, K., and Shimada, T. 2013. "Conceptual Design of Single-Stage Launch Vehicle with Hybrid Rocket Engine for Scientific Observation Using Design Informatics." Journal of Space Engineering 6 (1): 15-27.

[5] Watanabe, S., Chiba, Y., and Kanazaki, M. 2014. "A Proposal on Analysis Support System Based on Association Rule Analysis for Non-dominated Solutions." Presented at the 2014 IEEE Congress on Evolutionary Computation, Peking, China.

[6] Hikone, S., Hasegawa, T., and Nakagawa, I. 2010. "Regression Rate Characteristics and Combustion Mechanism of Some Hybrid Rocket Fuels.” Presented at AJCCP 2010, Miyazaki.

[7] Yuasa, S., Yamamoto, K., Hachiya, H., Kitagawa, K., and Owada, Y. 2001. "Development of a Small Sounding Hybrid Rocket with a Swirling-Oxidizer-Type Engine." Present at the 37th Joint Propulsion Conference and Exhibit, Salt Lake City, Utah.

[8] Gordon, S., and McBride, B. 1994. Computer Program for Calculation of Complex Chemical Equilibrium Compositions and Applications I. Analysis, NASA RP-1311.

[9] Japan Aerospace Exploration Agency. 2008. "The M-5 Rockets: From the Fifth to the Eighth Vehicle." JAXA-SP-07-023.

[10] Humble, R., Henry, G., and Larson, W. 1995. Space Propulsion Analysis and Design, Learning Solutions, New York.

[11] Flight Experiment Plan for 2nd Sounding Rocket in FY1996, ISAS/JAXA (in Japanese).

[12] Raymer, D. 2006. Aircraft Design: A Conceptual Approach (AIAA Education Series). 4th edition. USA: Amer. Inst. of Aeronautics.

[13] Kanazaki, M., and Seto, N. 2012. "Efficient Global Optimization Applied to Design and Knowledge Discovery of Supersonic Wing." Journal of Computational Science and Technology 6 (1): 1-15.

[14] Kanazaki, M., and Jeong, S. 2007. "High-Lift Airfoil Design Using Kriging based MOGA and Data Mining." Korea Society for Aeronautical and Space Sciences International Journal 8 (2): 28-36.

[15] Kohonen, T. 2000. Self-organizing Maps. Ney Nork: Springer.

[16] Vesant, J. 1999. "SOM Based Data Visualization Method." Intelligent-Data-Analysis 3: 111-26. 